\title{
ANÁLISE DA CONSISTÊNCIA ALIMENTAR E TEMPO DE DEGLUTIÇÃO EM CRIANÇAS COM PARALISIA CEREBRAL TETRAPLÉGICA ESPÁSTICA
}

\section{Analysis of alimentary consistency and deglutition time in children with spastic quadriplegic cerebral palsy}

\author{
Graziela Pacheco Vivone (1), Michelle Martins Mattos Tavares ${ }^{(2)}$, Renata de Salles Bartolomeu (3), \\ Kátia Nemr ${ }^{(4)}$, Ana Lúcia de Magalhães Leal Chiappetta ${ }^{(5)}$
}

\begin{abstract}
RESUMO
Objetivo: avaliar a consistência alimentar e o tempo de deglutição em crianças que apresentam Paralisia Cerebral Tetraplégica Espástica. Métodos: foram avaliadas 30 crianças entre 0 e 12 anos, com Paralisia Cerebral do tipo tetraplegia espástica, em acompanhamento fonoaudiológico, sendo utilizado um protocolo de avaliação nos padrões de deglutição. Resultados: os dados coletados mostraram a fase oral ineficiente ou ausente em 27 crianças (90\%) e a fase faríngea com anormalidades ou inadequações em 21 crianças (70\%). Mais da metade das crianças avaliadas apresentaram disfunção motora oral (DMO) entre moderada e grave. Em relação à consistência do alimento e os tempos de deglutição, as crianças com Função Motora Oral profundamente comprometida levam 14,2 vezes mais tempo para deglutir alimentos líquidos e 6,4 vezes mais para o alimento pastoso. Conclusão: diante do estudo realizado, é possível concluir que a maior dificuldade da criança que apresenta paralisia cerebral é a fase oral da deglutição e quanto maior a disfunção motora oral, maior o tempo gasto para deglutir os alimentos nas três consistências pesquisadas. As relações entre DMO e tempo de deglutição estão diretamente relacionadas.
\end{abstract}

DESCRITORES: Deglutição; Paralisia Cerebral; Alimentação

\section{INTRODUÇÃO}

A Paralisia Cerebral é uma desordem do tônus, do movimento e da postura de caráter não-progressivo devido a uma lesão que afeta o cérebro imaturo e interfere na maturação do Sistema Ner-

(1) Fonoaudióloga da Clínica Vivone; Especialista em Motricidade Orofacial pelo CEFAC - Saúde e Educação.

(2) Fonoaudióloga da Clínica Tavares; Especialista em Motricidade Orofacial pelo CEFAC - Saúde e Educação.

(3) Fonoaudióloga da Prefeitura Municipal de Volta Redonda; Especialista em Motricidade Orofacial pelo CEFAC - Saúde e Educação; Pós-graduada em Psicomotricidade pela Escola Superior de Ensino Helena Antipoff.

(4) Fonoaudióloga Responsável pelo serviço de Fonoaudiologia do Hospital Heliópolis; Doutora em Psicologia Social pela Universidade de São Paulo.

(5) Fonoaudióloga do Setor de Investigação em Doenças Neuromusculares da Universidade Federal de São Paulo; Doutora em Neurociências pela Universidade Federal de São Paulo. voso Central. Esta lesão provoca uma debilitação na coordenação da ação muscular com resultante incapacidade da criança em manter posturas e realizar movimentos normais. Qualquer agente capaz de lesar o encéfalo da concepção à primeira infância é considerado como causa da Paralisia Cerebral ${ }^{1,2}$. Os locais anatômicos de envolvimento, os graus de deficiência motora associada às disfunções e as causas são heterogênicos ${ }^{3}$.

Dentre seus diferentes tipos, está o espástico, que será abordado neste trabalho e que é caracterizado por aumento de tônus muscular global, hiperreflexia, déficits motores, entre outros ${ }^{4}$. Esta variedade é a mais freqüente e indica uma lesão fixa na parte motora do córtex cerebral. A criança com este tipo de desordem apresenta musculatura hipertônica, ou seja, estado de contração muscular aumentado mesmo estando em repouso, com grau variado de contratura ${ }^{5,6}$. Quanto ao comprometi- 
mento motor, a Paralisia Cerebral está dividida em tetraplegia, diplegia e hemiplegia ${ }^{7,8}$.

Tais desordens motoras podem acarretar alterações da movimentação e da postura, interferindo, assim, no desenvolvimento dos órgãos orofaciais e proporcionando o desempenho inadequado das funções de fala, alimentares, de deglutição, alterações articulatórias e alterações respiratórias ${ }^{9,10}$.

Nestas desordens neurológicas poderão ocorrer alterações em qualquer uma das fases da deglutição, causando uma disfagia neurogênica, e a Paralisia Cerebral é uma das mais importantes doenças neurológicas referidas ${ }^{11-13}$.

A dificuldade motora básica dessas crianças pode afetar a função motora oral, influenciando no desempenho das funções alimentares de sucção, mastigação e deglutição, desenvolvendo também alterações articulatórias e respiratórias, entendida como os aspectos motores e sensoriais das estruturas da cavidade oral e da faringe até a entrada do esôfago ${ }^{14}$.

As características do portador da Paralisia Cerebral Tetraplégico Espástico são consideradas fatores de risco para distúrbios alimentares: pela alteração motora da dinâmica orofaríngea; pela falta de compreensão do contexto alimentar e dificuldade na ação motora voluntária da fase oral, podendo alterar a seqüencialização da fase faríngea; e pela gravidade da aspiração traqueal ${ }^{15}$.

Quanto à dinâmica orofaríngea, os comprometimentos da fase oral são caracterizados pela incapacidade de controlar o alimento na boca. Estes podem ser tão graves que os problemas na fase faríngea podem ser pouco percebidos. Isso pode ocorrer pela dificuldade no vedamento labial, perda dos reflexos orais e da movimentação das partes anterior e dorsal da língua ${ }^{15}$.

Tendo em vista a importância da alimentação para a vida dos indivíduos e a dificuldade que as crianças com Paralisia Cerebral podem apresentar para deglutir, o objetivo deste estudo é analisar a fase oral da deglutição, relacionando a consistência dos alimentos, o tempo de deglutição e os reflexos motores orais nestas crianças.

\section{MÉTODOS}

Este estudo foi realizado em 30 crianças com faixa etária entre 2 e 12 anos, com Paralisia Cerebral Tetraplégica Espástica, que estão em atendimento fonoaudiológico. Houve crianças que apresentaram síndromes associadas à paralisia e níveis de desenvolvimento cognitivo variados, não sendo fatores de restrição ao estudo.

A classificação da Paralisia Cerebral de cada criança foi obtida junto ao laudo médico, após autorização dos pais (ou responsáveis) para essa pesquisa.

As crianças foram posicionadas sentadas, de forma a ficarem confortáveis em postura estável, sendo que algumas receberam ajuda adicional do responsável para controle da postura.

Para a realização do exame foi elaborado um protocolo de avaliação nos padrões da deglutição, o qual foi aplicado nas crianças sob as mesmas condições ${ }^{11}$ (Figura 1).

O material para aplicação do protocolo foi convencionado como sendo: $10 \mathrm{ml}$ de suco oferecido no copo, $10 \mathrm{~g}$ de iogurte de morango homogêneo oferecido na colher e 10 gramas de pão oferecido em pedaços pequenos, os quais foram ingeridos em sua totalidade e na ordem apresentada. No caso de recusa, o material não foi ministrado. Foi utilizado ainda cronômetro com medidas em minutos e segundos para se mensurar os tempos de deglutição de cada tipo de alimento.

\begin{tabular}{|c|c|}
\hline \multicolumn{2}{|c|}{ Protocolo de Avaliação Fonoaudiológica } \\
\hline \multicolumn{2}{|c|}{ 1. Reflexo de Mordida } \\
\hline ( ) Presente & ( ) Ausente \\
\hline \multicolumn{2}{|c|}{ 2. Captação do Bolo Alimentar } \\
\hline ( ) Presente & ( ) Ausente \\
\hline \multicolumn{2}{|c|}{ 3. Vedamento Labial } \\
\hline ( ) Presente & ( ) Ausente \\
\hline \multicolumn{2}{|l|}{ 4. Sialorréia } \\
\hline ( ) Presente & ( ) Ausente \\
\hline \multicolumn{2}{|l|}{ 5. Tragar o Copo } \\
\hline ( ) Presente & ( ) Ausente \\
\hline \multicolumn{2}{|c|}{ 6. Retirar da Colher } \\
\hline ( ) Presente & ( ) Ausente \\
\hline \multicolumn{2}{|c|}{ 7. Preparar o Bolo Alimentar } \\
\hline ( ) Presente & ( ) Ausente \\
\hline \multicolumn{2}{|c|}{ 8. Condução do Bolo Alimentar } \\
\hline ( ) Presente & ( ) Ausente \\
\hline \multicolumn{2}{|c|}{ 9. Mobilidade Laríngea } \\
\hline ( ) Presente & ( ) Ausente \\
\hline \multicolumn{2}{|c|}{ 10. Refluxo Nasal } \\
\hline ( ) Presente & ( ) Ausente \\
\hline \multicolumn{2}{|l|}{ 11. Engasgos } \\
\hline ( ) Presente & ( ) Ausente \\
\hline \multicolumn{2}{|c|}{ 12. Respiração Ruidosa Durante a Deglutição } \\
\hline ( ) Presente & ( ) Ausente \\
\hline
\end{tabular}

Figura 1 - Protocolo de avaliação fonoaudiológica 
A avaliação foi realizada com os usuários do Programa de Reabilitação em Comunidade, no Rio de Janeiro, em domicílio.

Durante a avaliação foram observadas as fases preparatória e oral, verificando se o paciente captou todo o alimento, se captou parcialmente ou assistematicamente, sendo considerado presente, e quando não captou o alimento, considerado ausente.

Foi verificado se há presença do vedamento labial anterior, ou se há escape de alimento, considerado presente, ou total falta de selamento labial, considerado ausente. No preparo do bolo foi utilizado alimento sólido. O paciente que conseguiu realizar a incisão ou trituração com os dentes ou quando realizada apenas movimentação pósteroanterior da língua em lugar da trituração, foi considerado presente. O paciente que não faz o uso de alimento sólido foi classificado como ausente.

$\mathrm{Na}$ fase faríngea foi avaliada a mobilidade laríngea, sendo presente na elevação laríngea e ausente na assimetria da mobilidade.

Foi analisada a presença ou ausência de reflexos motores orais.

O protocolo de investigação consta da identificação e do resultado da observação da presença dos seguintes comportamentos relacionados à função motora oral: reflexo de mordida, captação do bolo alimentar, vedamento labial, sialorréia, tragar o copo, retirar da colher, preparo do bolo alimentar (mastigar), condução, mobilidade faríngea, refluxo nasal, engasgos, respiração ruidosa durante a deglutição ${ }^{13}$.

A pesquisa foi realizada por uma investigadora $e$ observada pelas demais pesquisadoras, sendo que a análise dos dados e a pontuação dos resultados foram realizadas em consenso.

Para analisar os resultados obtidos foi elaborada uma escala, atribuindo-se valor 1 para comportamentos considerados presentes, 0 para comportamentos ausentes, aplicados a cada um dos itens avaliados (Figura 2).

Cada criança recebeu uma pontuação final que corresponde à soma dos valores dos 12 itens avaliados, de forma que a pontuação poderia variar entre 0 e 12 pontos, demonstrando o desempenho de cada criança, estando mais próxima do normal quanto mais próxima de 12 estiver do total.

Registrado os tempos gastos para deglutir cada um dos três tipos de alimentos, a partir da primeira introdução na boca, até seu esvaziamento na última deglutição. Fez-se comparação entre os tempos medianos de deglutição. As crianças foram reagrupadas conforme o grau da disfunção motora oral: leve, moderada, grave e profunda ou função motora oral normal (Figura 3).

\begin{tabular}{|l|c|c|}
\hline Função Motora Oral (FMO) & $\mathbf{1}$ & $\mathbf{0}$ \\
\hline Reflexo de Mordida & Ausente & Presente \\
\hline Captação do Bolo Alimentar & Presente & Ausente \\
\hline Vedamento Labial & Presente & Ausente \\
\hline Sialorréia & Ausente & Presente \\
\hline Tragar o Copo & Presente & Ausente \\
\hline Retirar da Colher & Presente & Ausente \\
\hline Preparar o Bolo Alimentar & Presente & Ausente \\
\hline Condução & Normal & Anormal \\
\hline Mobilidade Laríngea & Presente & Ausente \\
\hline Refluxo Nasal & Ausente & Presente \\
\hline Engasgos & Ausente & Presente \\
\hline $\begin{array}{l}\text { Respiração Ruidosa Durante } \\
\text { a Deglutição }\end{array}$ & Ausente & Presente \\
\hline
\end{tabular}

Figura 2 - Itens considerados na função motora oral e valores atribuídos

\begin{tabular}{|l|c|}
\hline Função Motora Oral & Valores \\
\hline Normal & 12 \\
\hline Leve & $11-8$ \\
\hline Moderada & $7-5$ \\
\hline Grave & $4-2$ \\
\hline Profunda & $1-0$ \\
\hline
\end{tabular}

Figura 3 - Classificação da alteração da função motora oral e valores atribuídos

Para cada um dos grupos, levou-se em consideração o tempo mediano gasto na deglutição de cada uma das consistências avaliadas. Desta forma, analisou-se a influência da disfunção motora oral e foram relacionadas as três consistências de alimentos nos tempos de deglutição.

Este trabalho foi analisado pelo Comitê de Ética em Pesquisa do CEFAC - Saúde e Educação - sendo aprovado com o número de protocolo 121/06.

Os dados foram apresentados na forma descritiva com freqüências absoluta $(\mathrm{N})$ e relativas (\%) em forma tabular.

\section{RESULTADOS}

A avaliação da fase oral da deglutição das 30 crianças com Paralisia Cerebral Tetraplégica Espástica demonstrou ser ineficiente ou ausente em 27 sujeitos (90\%). Em apenas 3 crianças $(10 \%)$ a deglutição foi classificada como eficiente (Tabela 1). Sendo assim, a grande maioria apresenta a fase oral inadequada. 
Tabela 1 - Dados referentes à avaliação da fase oral da deglutição das 30 crianças com Paralisia Cerebral

\begin{tabular}{lcc}
\hline Fase Oral & $\begin{array}{c}\text { Número de } \\
\text { Crianças (N) }\end{array}$ & $\%$ \\
\hline Eficiente & 3 & 10 \\
Ineficiente & 13 & 43,4 \\
Ausente & 14 & 46,6 \\
Total & 30 & 100 \\
\hline
\end{tabular}

$\mathrm{Na}$ avaliação da fase faríngea da deglutição das 30 crianças com paralisia cerebral, observam-se anormalidades ou inadequações em 21 delas $(70 \%)$. O engasgo foi o tipo de alteração mais observado, ocorrido em 23 crianças (76\%). Outras inadequações e respectivas ocorrências estão citadas na Tabela 2. Observa-se que em uma mesma criança foi constatada mais de uma dificuldade.

Tabela 2 - Dados referentes a alterações da fase faríngea da deglutição das 30 crianças com Paralisia Cerebral

\begin{tabular}{lcc}
\hline Fase Faríngea & Espástico(N) & $\%$ \\
\hline Mobilidade Laríngea & 5 & 16,6 \\
Reduzida & 10 & 33,3 \\
Refluxo Nasal & 23 & 76,6 \\
Engasgos & 30 & 100 \\
$\begin{array}{l}\text { Respiração Ruidosa } \\
\text { durante a Deglutição }\end{array}$ & 30 \\
\hline
\end{tabular}

Outra análise compara 8 itens da função motora oral (FMO) que foram associados ao grau da disfunção motora oral (DMO). Os tempos de deglutição foram associados à FMO normal, levemente, moderadamente, gravemente e profundamente comprometida.

A capacidade de controlar os lábios evitando o escape do bolo alimentar para fora da cavidade oral durante a deglutição foi o item de menor ocorrência. Já a habilidade de captação do preparo para o bolo alimentar foi a mais adequada (Tabela 3 ).

A condução anormal ocorreu associada ao reflexo de mordida em $43 \%$ dos casos, a presença da capacidade de tragar do copo em $57 \%$, a capacidade de retirar o alimento da colher em $28 \%$. Já associada à ausência do controle labial em $96 \%$ dos casos e a presença de sialorréia em $93 \%$. (Tabela 4).
Tabela 3 - Distribuição da amostra segundo a presença de FMO

\begin{tabular}{lcc}
\hline FMo & $\begin{array}{c}\text { 30 crianças } \\
\text { (N) }\end{array}$ & $\%$ \\
Reflexo de Mordida & 14 & 24 \\
Captação do Bolo & 16 & 53 \\
Alimentar & 2 & 9 \\
Vedamento Labial & 28 & 90 \\
Sialorréia & 14 & 44 \\
Tragar o Copo & 8 & 28 \\
Retirar da Colher & 16 & 53 \\
Preparar para o Bolo & 6 & 19 \\
Alimentar & & \\
Condução &
\end{tabular}

Tabela 4 - Condução anormal e a ocorrência dos itens pesquisados da FMO

\begin{tabular}{lcc}
\hline FMO & $\begin{array}{c}\text { Condução } \\
\text { anormal (28) }\end{array}$ & $\%$ \\
\hline Reflexo de Mordida & 12 & 43 \\
Captação do Bolo & 15 & 54 \\
Alimentar & 1 & 4 \\
Vedamento Labial & 26 & 93 \\
Sialorréia & 15 & 57 \\
Tragar o Copo & 7 & 28 \\
Retirar da Colher & 15 & 54 \\
Preparar para o Bolo & & \\
\hline
\end{tabular}

A seguir está apresentada a classificação da FMO das crianças, conforme pontuação dada a cada item da FMO. $100 \%$ das crianças demonstraram algum grau de disfunção motora oral variando entre leve e profunda.

Distribuição Segundo Classificação da FMO

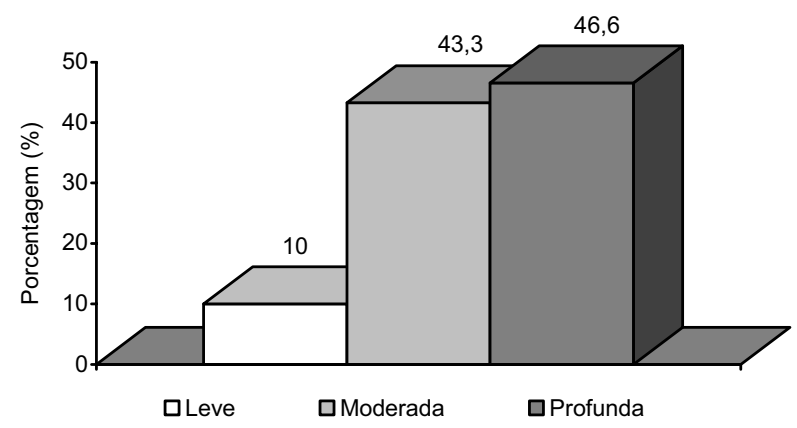

Figura 4 - Itens considerados na função motora oral (FMO) e os valores atribuídos 


\section{Tabela 5 - FMO, consistência do alimento e tempos medianos de deglutição em segundos por grupo}

\begin{tabular}{lccc}
\hline DMO & Líquido & Pastoso & Sólido \\
\hline Leve & 92 & 101,5 & 238 \\
Moderada & 187 & 173 & 310 \\
Grave & 352,5 & 264 & 506 \\
\hline
\end{tabular}

As relações entre FMO e tempo de deglutição são mostradas na Tabela 5 e revelam que quanto maior a disfunção motora oral, maior o tempo gasto para realizar a deglutição em cada uma das três consistências de alimento, sugerindo que DMO e tempo de deglutição estão diretamente relacionados.

Das crianças, 46,6\% apresentaram disfunção motora oral grave. Em relação à consistência do alimento e os tempos de deglutição, as crianças com FMO profundamente comprometida levam 14,2 vezes mais tempo para deglutir alimentos líquidos e 6,4 vezes mais para o alimento pastoso do que as crianças normais. Nenhuma das crianças com disfunção motora oral profunda deglutiu a consistência sólida. Das crianças com disfunção motora oral grave, apenas $10 \%$ deglutiram a consistência sólida, as quais levaram 4,9 vezes mais tempo para deglutir o sólido que tempo normal. $O$ alimento pastoso foi aquele em que os tempos de deglutição mais se aproximaram entre os dois grupos e o líquido, a consistência que se observou maior diferença dos tempos medianos de deglutição entre os dois grupos estudados. O alimento sólido foi a consistência com menor aceitação do grupo (Tabela 5).

\section{DISCUSSÃO}

No presente estudo observou-se que a maioria das crianças com Paralisia Cerebral Tetraplégica Espástica apresenta fase oral ineficiente ou ausente, assim como, anormalidades ou inadequações na fase faríngea da deglutição. Tais achados comprovam ser a paralisia cerebral uma das mais importantes doenças neurológicas referidas que pode ser acompanhada com disfagia orofaríngea ${ }^{10,13}$.

A disfagia identificada nesses pacientes estava relacionada ao processo de mastigação propriamente dito e ao posicionamento e direcionamento do bolo para região posterior da cavidade oral. Este estudo vem comprovar que a criança com Paralisia Cerebral Tetraplégica Espástica apresenta alterações que interferem diretamente na deglutição, como, por exemplo, a falta de vedamento labial e as alterações posturais, fazendo-se necessária uma avaliação clínica detalhada, analisando todas as informações de forma a direcionar o planejamento terapêutico.

As habilidades e os comportamentos considerados mais maduros, como, vedamento labial, condução, retirar da colher, tragar o copo, captação e preparação do bolo alimentar ocorreram com menor freqüência. Estes achados corroboram outras pesquisas, em que apontam como dificuldades a ausência de lateralização da língua, falta de controle do alimento na boca e falta de controle da cabeça e do tronco, os maiores problemas na alimentação da criança com paralisia cerebral ${ }^{13,14}$.

Pôde-se observar ausência do controle labial em $96 \%$ dos casos e DMO variando entre leve e profunda, em $100 \%$ das crianças. Estes achados enfatizam a importância do vedamento labial para a deglutição, pois, quando eficiente, mantém a pressão intra-oral que auxilia, juntamente com o movimento propulsor da língua, a direcionar o bolo alimentar para faringe ${ }^{10}$. Verificou-se a condução anormal dos alimentos em 28 crianças, isto explica que quando há ineficiência no vedamento, esse mecanismo pressórico acaba sendo afetado e, conseqüentemente, o transporte do alimento, a captação do alimento da colher, ou mesmo a tentativa de beber um líquido junto ao copo, é dificultosa, pois o paciente não é capaz de unir os lábios em razão do prejuízo motor ${ }^{15}$.

Os resultados encontrados apontam a DMO como uma das causas que mais comprometem a alimentação destas crianças. Esta inadequação justifica que a mastigação é deficitária quanto à tendência desses pacientes de realizarem o "amassamento" do alimento com movimentos pósteroanteriores de língua e movimentos verticais de mandíbula, mostrando que essa falta de controle oral caracteriza uma ineficiência na mastigação ${ }^{14}$. Tais dificuldades decorrem da presença de rigidez na musculatura orofacial dos espásticos ${ }^{15}$.

Verificou-se neste estudo que a observação da atividade reflexa anormal e da sialorréia tem sido objeto de muitos trabalhos sobre distúrbio da deglutição em crianças com paralisia cerebral, uma vez que acarretam transtornos na FMO e espelham o funcionamento imaturo do aparelho estomatognático. Da mesma forma, as habilidades de conter o bolo alimentar, tragar líquidos, retirar alimento da colher, mastigar e deglutir normalmente relacionase diretamente com a especialização dos movimentos do trato aerodigestivo, desenvolvimento do sistema estomatognático e amadurecimento do sistema nervoso central. Essas habilidades tornam possível a manutenção da boa nutrição e o crescimento dos indivíduos ${ }^{16}$. Por essa razão, escolheuse observar esses aspectos da FMO. 
Das três consistências pesquisadas, o alimento líquido foi o que mostrou maiores diferenças de tempo para ser deglutido e o pastoso, o menor. A deglutição do alimento sólido foi o de menor aceitação que as outras consistências, mas quando ocorre, a diferença entre o tempo gasto é menor que para o alimento líquido. A consistência pastosa deve ser a mais indicada para crianças com disfunção motora oral e desnutrição, uma vez que é mais fácil de ser manipulada e pode reduzir a tosse e a aspiração, facilitar a alimentação e a nutrição da criança. Assim como o uso do alimento sólido associado à estimulação motora oral para o tratamento de crianças com paralisia cerebral, tendo como objetivo diminuir o tempo de trânsito faríngeo, diminuir resíduo e a necessidade de múltiplas deglutições para limpar o conteúdo da faringe.

Uma vez que os problemas nutricionais são desafios para quem atua com crianças com paralisia cerebral, faz-se necessário compreender a condição de saúde destas. Desta forma, o fonoaudiólogo deve contemplar a questão da saúde da criança com uma visão integral, observando a função motora oral dentro da perspectiva da boa nutrição e hidratação da criança por ele assistida. Isso equivale a dizer ter uma visão funcional da deglutição, observando se a capacidade motora de transportar o bolo alimentar e a eficácia para manter a boa nutrição da criança e colaborar no seu desenvolvimento, objetivo principal desse processo.

Autores realizaram estudos sobre a análise qualitativa da deglutição, no que diz respeito às alterações de postura e movimento, quanto à capacidade de reduzir, formar e propelir o bolo alimentar, bem como o escape prematuro, penetração e aspiração de alimentos nas vias aéreas, entre outras, comprovando ser este estudo de grande utilidade clínica, pois fornecem sustentação teórica para a prática diária com indivíduos que sofrem de problemas dessa natureza.

Foi com essa visão que se desenvolveu este trabalho, com o intuito de discutir questões relativas ao tempo de deglutição dessas crianças, e em última instância, uma reflexão acerca do tempo real gasto por elas durante as refeições diárias. A relevância deste questionamento baseia-se na necessidade de uma visão realista e objetiva em relação à deglutição, para preconizarem-se melhores procedimentos e condutas a respeito da alimentação das mesmas.

Sendo assim, os parâmetros quantitativos, como o tempo do transporte do bolo em cada etapa do processo e o tempo gasto nas refeições, podem ser parâmetros igualmente importantes ${ }^{17}$. E a análise quantitativa deve ser considerada, especialmente para as crianças que possuem o distúrbio da fun- ção motora oral em conjunto com má nutrição, crescimento linear, perda de peso ou baixa imunidade, entre outras. Enfim, neste estudo pôde-se comprovar que a utilização de uma consistência de alimento de mais fácil deglutição deve ser preconizada nesses casos, antes que outras técnicas mais invasivas sejam necessárias, como por exemplo, a gastrostomia ${ }^{18,19}$.

Algumas correlações importantes foram feitas entre os comportamentos observados. A ocorrência de uma habilidade mais madura geralmente é acompanhada de outras. A correlação entre a deglutição normal e a grande porcentagem de crianças com ausência do reflexo de mordida, capacidade de retirar o alimento da colher, capacidade de tragar líquido e a possibilidade da mastigação podem fornecer importante material de suporte a prática terapêutica. A prescrição de programas de tratamento incentivando o surgimento dessas habilidades possivelmente levará a ganhos quantitativos e qualitativos em relação à deglutição dessas crianças, melhora de sua condição nutricional e qualidade de vida.

Outras pesquisas também mostram que uma idéia do tempo despendido pela família para alimentar as suas crianças é ponto importante para que se decida a respeito das consistências de alimentos mais indicadas para cada criança, a forma de alimentá-las e a freqüência ${ }^{20}$.

Os achados da presente pesquisa estão condizentes com estas observações, pois indicam a existência de uma grande diferença entre o tempo gasto para alimentar crianças sem distúrbios neurológicos e crianças com paralisia cerebral. Observou-se que as crianças com paralisia cerebral podem levar até 14,2 vezes mais tempo para deglutir. Outros autores encontram um dado bastante semelhante a este e declaram que as crianças podem gastar até 15 vezes mais tempo e levar até 7 horas por dia se alimentando, fato este que pode levar as famílias à frustração frente à dificuldade de alimentar essas crianças e ao estresse, colaborando para diminuição de oferta de alimento as mesmas e, conseqüente, má nutrição. ${ }^{21}$

Estudos anteriores já indicam que as famílias tendem a gastar um tempo muito menor que o necessário para alimentar essas crianças, o que pode contribuir para uma diminuição da quantidade da ingesta deglutida e, usualmente, aumentar as chances de má nutrição e hidratação. ${ }^{22}$

Os resultados sugerem a necessidade de monitorização das crianças com paralisia cerebral, especialmente as tetraplégicas, no que se refere às questões da deglutição e nutrição. O enorme tempo gasto durante as refeições, a reduzida quantidade de alimento, a disfunção motora oral, o crescimento e o aumento no gasto de energia, podem contribuir 
para uma estagnação do crescimento e prejuízos no desenvolvimento global da criança.

\section{CONCLUSÃO}

Diante do estudo realizado, é possível concluir que a maior dificuldade da criança que apresenta Paralisia Cerebral consiste na fase oral inadequada, assim como, anormalidades ou inadequações na fase faríngea.
Por meio da aplicação do protocolo de avaliação dos padrões de deglutição em um grupo de 30 crianças, com Paralisia Cerebral Tetraplégica Espástica, obteve a conclusão de que quanto maior a disfunção motora oral, maior o tempo gasto para deglutir os alimentos nas respectivas consistências pastoso, líquido e sólido.

As relações entre DMO e tempo de deglutição estão diretamente relacionadas.

\begin{abstract}
Purpose: to evaluate the alimentary consistency and the deglutition time in children with Cerebral Palsy. Methods: 30 children between 0 and 12 years, with Cerebral Paralysis of the quadriplegic spastic type been evaluated, that were under speech pathology follow-up. Results: the collected data showed that the verbal phase demonstrated to be inefficient or absent in the majority of the children, as well as in the pharyngeal phase of deglutition. More of the half of the evaluated children have shown between moderate to acute verbal motor dysfunction. In relation to the consistency of food and deglutition times, the children with deeply compromised OMF (Oral Motor Function) took more 14.2 times to swallow liquid foods and more 6.4 times for the pasty food. Conclusion: before the study, it is possible to conclude that the major difficulty of the child with cerebral paralysis is the verbal phase of deglutition and as bigger the verbal motor dysfunction, the bigger is the time elapsing for swallowing foods in the three studied consistencies. The relations between OMD (Oral Motor Dysfunction) and deglutition time are directly related.
\end{abstract}

KEYWORDS: Deglutition; Cerebral Palsy; Feeding

\section{REFERÊNCIAS}

1. Leitão A. Paralisia cerebral: diagnóstico, terapia e reabilitação. São Paulo: Atheneu; 1983.

2. Barbosa RC, Santiago WO. Perfil do atendimento de pacientes portadores de paralisia cerebral em instituições filantrópicas do município de Campinas. Rev Fono Atual. 2003; 6(23):19-25.

3. Tecklin JS. Fisioterapia pediátrica. Porto Alegre: Artmed; 2002.

4. Buchkols DW, Robbins J. Neurologic diseases affecting oropharyngeal swallowing. In: Peralman A, Schulze-Delriev K. Deglution and its disorders. San Diego: Singular; 1997

5. Schwartzman JS. Paralisia cerebral. Temas sobre desenvolvimento. 3(13):4-21; 1993.

6. Madalozzo D, Mitre IE. Análise da fase oral da deglutição de adultos com paralisia cerebral espástica e tetraplegia. Rev CEFAC. 2004; 6(4):345-9.

7. Bobath K. Uma base neurofisiológica para tratamento da paralisia cerebral. São Paulo: Manole; 2000.

8. Rosemberg S. Neuropediatria. São Paulo: Sarvier; 1998.
9. Seacero LF, Guedez ZCF. Avaliação dos órgãos fonoarticulatórios e aspectos odontológicos em crianças portadoras de paralisia cerebral com espasticidade e quadriplegia. In: Marchesan I, Zorzi J. Anuário CEFAC de fonoaudiologia 1999/2000. Rio de Janeiro: Revinter; 2000. p.251-7.

10. Furkin AM, Santini CS. Disfagias Orofaríngeas. Barueri: Pró-Fono; 2004.

11. Giubbina CA, Ferreira VJA. A deglutição na paralisia cerebral. Rev CEFAC. 2002; 4(1):29-34.

12. Gomes AM, Novaes CRG, Ferreira VJA. Disfagia orofaríngea neurogênica: principais fatores determinantes da recusa alimentar no paciente portador de paralisia cerebral do tipo espástico institucionalizado. Rev CEFAC. 2002; 4(1):25-8.

13. Seacero LF, Guedes ZCF. Aspectos da deglutição em crianças com paralisia cerebral espástica. Rev Fono Atual. 1999; 10:20-4.

14. Cesa CC, Ecco CT, Bersch R, Chiappetta ALML. Funções do sistema estomatognático e reflexos motores orais em crianças com encefalopatia crônica infantil do tipo quadriparesia espástica. Rev CEFAC. 2004; 6(2):158-63.

15. Furkim AM, Behlau MS, Weckx LLM. Avaliação clínica e videofluoroscópica da deglutição em 
crianças com paralisia cerebral tetraparética espástica. Arq Neuro-Psiquiatr. 2003; 61(3A):611-6.

16. Mueller H. Alimentação. In: Finnie NA, organizador. O manuseio em casa da criança com paralisia cerebral. 2. ed. São Paulo: Manole; 1980. p.131-53.

17. Limbrock GJ, Hoyer H, Scheying H. Drooling, chewing and swallowing dysfunctions in children with cerebral palsy:treatment according to Castillo-Morales. ASCD J Dent Child. 1990; 57(6):445-51.

18. Arvedson J, Rogers B, Brodsky J. Anatomy, embriology and physiology. In: Arvedson JC, Brodsky L, organizador. Pediatric swallowing and feeding: assessment and management. San Diego: Singular Publishing Group ; 1993.
19. Dantas RO, Dodds WJ. Influência da viscosidade do bolo alimentar deglutido na motilidade da faringe. Arq Gastroenterol. 1990; 27(4):164-8.

20. Amundson JA, Sherbondy A, Van Dyke DC, Alexander R. Early identification and treatment necessary to prevent malnutrition in children and adolescents with severe disabilities. J Am Diet Ass. 1994; 94(8):880-3.

21. Bosma JF. Development and impairments of feeding in infancy and childhood. In: Groher ME. Dysphagia: diagnosis and management. 3. ed. Butterworth-Heinemann. 1997. p.131-67.

22. Morton RE, Bonas R, Fourie B, Minford J. Videofluoroscopy in the assessment of feeding disorders of children with neurological problems. Develop Med Child Neurol. 1993; 35(5):388-95. 